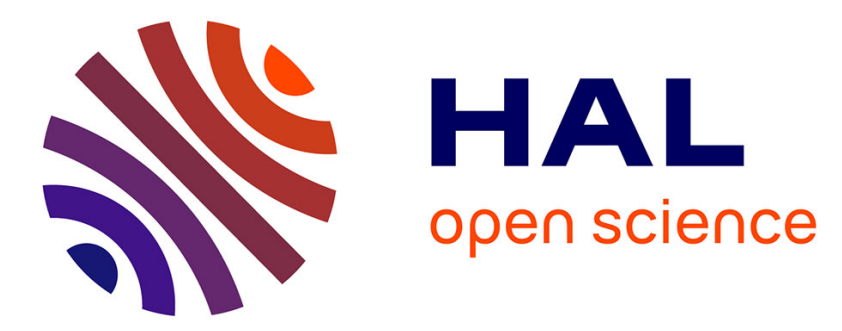

\title{
67P/Churyumov-Gerasimenko active areas before perihelion identified by GIADA and VIRTIS data fusion
}

A. Longobardo, V Della corte, S. Ivanovski, G. Rinaldi, V. Zakharov, A. Rotundi, F. Capaccioni, M. Fulle, G. Filacchione, E. Palomba, et al.

\section{- To cite this version:}

A. Longobardo, V Della corte, S. Ivanovski, G. Rinaldi, V. Zakharov, et al.. 67P/ChuryumovGerasimenko active areas before perihelion identified by GIADA and VIRTIS data fusion. Monthly Notices of the Royal Astronomical Society, 2019, 483 (2), pp.2165-2176. 10.1093/mnras/sty3244 . hal-02396715

\section{HAL Id: hal-02396715 \\ https://hal.science/hal-02396715}

Submitted on 1 Mar 2022

HAL is a multi-disciplinary open access archive for the deposit and dissemination of scientific research documents, whether they are published or not. The documents may come from teaching and research institutions in France or abroad, or from public or private research centers.
L'archive ouverte pluridisciplinaire HAL, est destinée au dépôt et à la diffusion de documents scientifiques de niveau recherche, publiés ou non, émanant des établissements d'enseignement et de recherche français ou étrangers, des laboratoires publics ou privés.

\section{(c)(1)}

Distributed under a Creative Commons Attribution| 4.0 International License 


\title{
67P/Churyumov-Gerasimenko active areas before perihelion identified by GIADA and VIRTIS data fusion
}

\author{
A. Longobardo, ${ }^{1,2 \star}$ V. Della Corte ${ }^{\oplus},{ }^{2,1}$ S. Ivanovski, ${ }^{1,2}$ G. Rinaldi ${ }^{\oplus},{ }^{1,2}$ V. Zakharov ${ }^{1}$ \\ A. Rotundi, ${ }^{2,1}$ F. Capaccioni, ${ }^{1}$ M. Fulle, ${ }^{3}$ G. Filacchione ${ }^{\odot},{ }^{1}$ E. Palomba,,${ }^{1,4}$ \\ P. Palumbo, ${ }^{2,1}$ M. T. Capria ${ }^{\odot},{ }^{1,2}$ F. Tosi ${ }^{\oplus},{ }^{1}$ A. Raponi ${ }^{\odot},{ }^{1}$ M. Ciarniello, ${ }^{1}$ \\ D. Bockelee-Morvan, ${ }^{5}$ S. Erard,${ }^{5}$ C. Leyrat, ${ }^{5}$ F. Dirri ${ }^{1}$ and \\ the GIADA and VIRTIS Teams ${ }^{1}$ \\ ${ }^{1}$ INAF-IAPS, via Fosso del Cavaliere 100, I-00133 Rome, Italy \\ ${ }^{2}$ Università di Napoli 'Parthenope', DIST, Centro Direzionale Isola C4, I-80143 Naples, Italy \\ ${ }^{3}$ INAF-OATS, Via G.B. Tiepolo 11, I-34143 Trieste, Italy \\ ${ }^{4}$ ASI-SSDC, via del Politecnico snc, I-00133 Rome, Italy \\ ${ }^{5}$ LESIA, Observatoire de Paris/CNRS/Université Pierre et Marie Curie/Université Paris-Diderot, F-92195 Meudon, France
}

Accepted 2018 November 23. Received 2018 November 2; in original form 2018 June 2

\begin{abstract}
We characterized 67P/Churyumov-Gerasimenko's cometary activity during its inbound arc before perihelion (2014 August-2015 January). We focused on the geomorphological regions of the Northern hemisphere observed by the ESA/Rosetta space probe during this time period. The GIADA dust detector characterized the physical properties of the fluffy and compact particles ejected from the nucleus; the VIRTIS imaging spectrometer detected exposed water ice.

We identified specific cometary active areas by adopting the following GIADA and VIRTIS indicators:

(a) The decrease with increasing temperature of the centre of the $3.2 \mu \mathrm{m}$ absorption band and of the spectral slope between 1.1 and $1.9 \mu \mathrm{m}$, as measured by VIRTIS, which are both possible effects of water ice exposition.

(b) The number of fluffy and compact particles ejected from a specific region, which we determined by tracing dust particles in the coma, as detected by GIADA, back down to the nucleus.

The correlation between GIADA and VIRTIS indicators led us to conclude that dust emission causes exposition of water ice on the surface of the nucleus, and fluffy and compact particles have common ejection regions; their lack of correlation in the coma is due to spread induced by their different velocities.

We find that the most active regions are located in the neck (Hapi and Seth) and in the body (Ash, Babi, and Aten) of the comet. These regions are also the ones most illuminated during the considered observation period, indicating that insolation is the main, if not only, driver of cometary activity.
\end{abstract}

Key words: instrumentation: detectors - methods: data analysis - techniques: imaging spectroscopy .

\section{INTRODUCTION}

The ESA/Rosetta spacecraft was launched in 2004 and orbited comet 67P/Churyumov-Gerasimenko (hereafter 67P) from 2014
August until 2016 September, escorting it during its perihelion, which occurred on 2015 August 13.

The nucleus of 67P is bi-lobate shaped (Sierks et al. 2015), on which four macro-regions have been defined, i.e. head, bottom, body, and neck. The images provided by the OSIRIS (Optical, Spectroscopic, and Infrared Remote Imaging System) camera (Keller et

^E-mail: andrea.longobardo@inaf.it 
al. 2007), have defined geomorphological regions (El Maary et al., 2015, 2016) in each macro-region.

The VIRTIS (Visual InfraRed and Thermal Imaging Spectrometer) imaging spectrometer (Coradini et al. 2007) revealed a dark nucleus surface, with an average geometric albedo of $0.062 \pm 0.002$ (Ciarniello et al. 2015). This surface is composed of dark materials: an unidentified spectrally featureless component (Capaccioni et al. 2015; hypothesized iron sulphides) and organics, carrier of the broad absorption band, centred at approximately $3.2 \mu \mathrm{m}$ (Capaccioni et al. 2015). Even if the nucleus surface is mostly dehydrated, water ice could be observed in the neck macro-region as the result of a diurnal water cycle (De Sanctis et al. 2015) or in localized patches, corresponding to exposed fresh material (Barucci et al. 2016; Filacchione et al. 2016a). Regardless of its origin, water ice exposure is a transient phenomenon driven by cometary activity and evolutionary processes, as confirmed by: (i) 67P surface spectra seasonal variations (Filacchione et al. 2016b; Ciarniello et al. 2016); (ii) temporal evolution of icy patches (Raponi et al. 2016); and (iii) carbon dioxide ice seasonal exposure (Filacchione et al. 2016c). Spectral variations associated with water ice are: (a) $3.2 \mu \mathrm{m}$ band deepening and shortward shift (e.g. De Sanctis et al. 2015); (b) visible $(0.5-0.8 \mu \mathrm{m})$ and near-infrared $(1-2 \mu \mathrm{m})$ spectral slope flattening (Filacchione et al. 2016b); and (c) appearance of 1.5 and $2.0 \mu \mathrm{m}$ absorption bands (only for crystalline ice; Filacchione et al. 2016b). In the inbound arc up to perihelion, the average trend in $3.2 \mu \mathrm{m}$ band is a shortward shift at increasing temperature, suggesting the exposition of water ice $(<0.1$ per cent) possibly due to cometary activity (Longobardo et al. 2017a). However, due to the small spectral shift observed, which is comparable to the VIRTIS spectral sampling, this conclusion cannot be considered definitive.

67P dust ejection has been studied by means of the GIADA (Grain Impact Analyser and Dust Accumulator) dust detector (Colangeli et al. 2007; Della Corte et al. 2014). GIADA detected mm-sized dust particles, belonging to two families (Fulle et al. 2015; Rotundi et al. 2015): compact particles $(0.03-1 \mathrm{~mm}$ in size) and fluffy particles $(0.2-2.5 \mathrm{~mm}$ in size). Fluffy particles are actually fragments of single pristine fluffy agglomerates (hereafter, parent particles), and their fragmentation is induced by the spacecraft potential (Fulle et al. 2015). Fluffy and compact particles do not appear to be spatially correlated in 67P's coma, and only compact particles ejection seems to be linked to solar illumination (Della Corte et al. 2015). Dust activity changes along 67P's orbit in terms of ejection rate (Della Corte et al. 2015, 2016a) and size distribution (Fulle et al. 2016).

This work provides further insights on cometary pre-perihelion activity by studying dust ejection rates and compositional variations in 67P's Northern hemisphere, i.e. the hemisphere observed in the inbound arc. Our study combines the GIADA and VIRTIS data sets in a synergistic way, as well as the related geometric information.

GIADA and VIRTIS data and high-level products are described in Sections 2 and 3, respectively. Data analysis is presented in Section 4, the results are shown in Section 5 and the interpretation is discussed in Section 6. Finally, the study's conclusions are presented in Section 7.

\section{DATA}

The VIRTIS imaging spectrometer consists of two optical heads: VIRTIS-H is a high-spectral resolution $(3 \mathrm{~nm})$ spectrometer covering the wavelength interval 2-5 $\mu \mathrm{m}$, while VIRTIS-M has imaging capabilities and works in the $0.2-5.1 \mu \mathrm{m}$ spectral range, covered by a visible $\left(0.2-1 \mu \mathrm{m}\right.$, spectral sampling $1.8 \mathrm{~nm}$ band $\left.^{-1}\right)$ and an infrared (1-5.1 $\mu \mathrm{m}$, spectral sampling $9.5 \mathrm{~nm} \mathrm{band}^{-1}$ ) channel, re- spectively. We analysed the VIRTIS-M infrared spectra, calibrated in radiance factor $\mathrm{I} / \mathrm{F}$ after removing thermal emission in the $3-5 \mu \mathrm{m}$ range (Raponi 2014).

The GIADA dust detector consists of three subsystems: (a) the grain detection system (GDS), a laser curtain with photodiodes; (b) the impact sensor (IS), a plate connected to piezoelectric sensors; and (c) the MicroBalance System (MBS), five quartz crystal microbalances facing in different directions. GDS and IS detect individual dust particles, measuring their speed (sensitivity $0.2 \mathrm{~m} \mathrm{~s}^{-1}$ ) and their momentum (sensitivity $10-10 \mathrm{~kg} \mathrm{~m} \mathrm{~s}^{-1}$ ), respectively, whereas MBS measures the cumulative dust mass flux (sensitivity $1.56 \mathrm{ng} \mathrm{cm}^{-2}$ ). Mass can be retrieved for dust particles detected both by GDS and IS subsystems (i.e. GDS + IS detections). In this study, we consider individual particle detections; therefore, we used GDS and IS data, not MBS measurements.

We used GIADA and VIRTIS data acquired from 2014 August 1 to 2015 January 9, specifically Medium-Term Planning (MTP) observations from MTP006 to MTP011. The VIRTIS data set contains 3.2 million spectra acquired at incidence and emission angles less than $70^{\circ}$, to avoid residual photometric effects observed at larger angles (Longobardo et al. 2017a). The spectra selection, since our analysis focuses on the $3.2 \mu \mathrm{m}$ band, excludes those in which the depth of this band is less than 0.04 , i.e. the depth value corresponding to the detector readout noise. No selection was made on heliocentric distance and local time. The GIADA data set, acquired in continuous mode during the indicated period, includes GDS-only, IS-only and GDS + IS for a total of approximately 800 detections. In the case of two simultaneous GDS measurements, we considered the following: (i) only the speed measured in the GDS + IS detection, if available; and (ii) the average of the two measured speeds.

\section{TOOLS}

\subsection{Geomorphological regions}

To study the cometary activity from each geomorphological region in 67P's Northern hemisphere, we used the geomorphological classification proposed by Thomas et al. (2015) and El-Maarry et al. (2015), as reference, including the 19 regions shown in Fig. 1.

\subsection{VIRTIS}

According to Longobardo et al. (2017a) the $3.2 \mu \mathrm{m}$ band centre shortward shift, observed at increasing surface temperature with respect to 67P's average, may indicate the exposition of a small

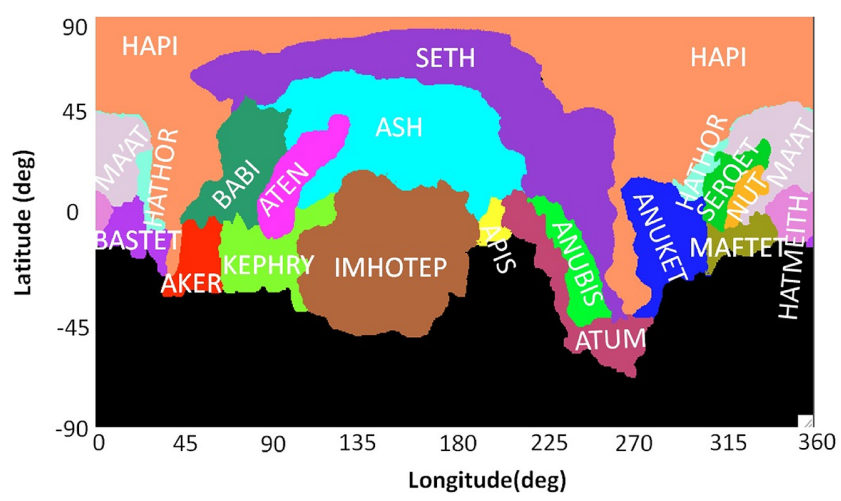

Figure 1. Cylindrical map of 67P/CG's 19 geomorphological regions as defined by El-Maarry et al. (2015) and Thomas et al. (2015). 


\section{$3.2 \mu \mathrm{m}$ band centre shift}

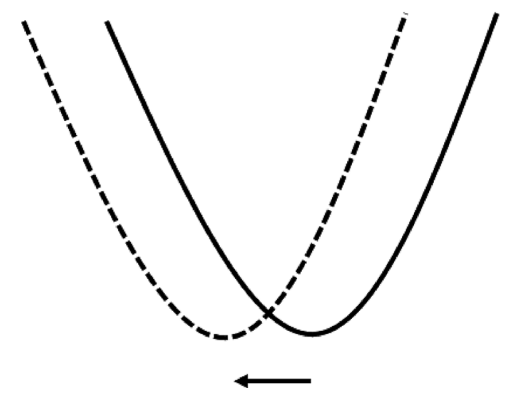

Infrared slope decrease

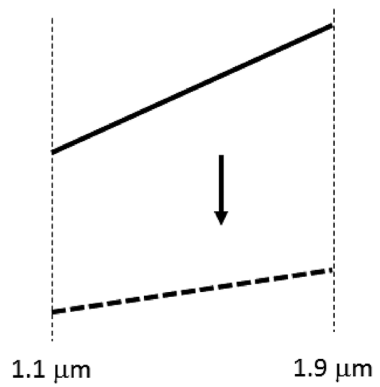

Low temperature spectrum

-_-n. High temperature spectrum

Figure 2. Schematic representation of spectral changes expected from exposition of water ice, due to increasing cometary activity at temperature higher than the 67P's average. The $3.2 \mu \mathrm{m}$ band centre would shift towards shorter wavelengths, whereas the infrared slope would decrease.

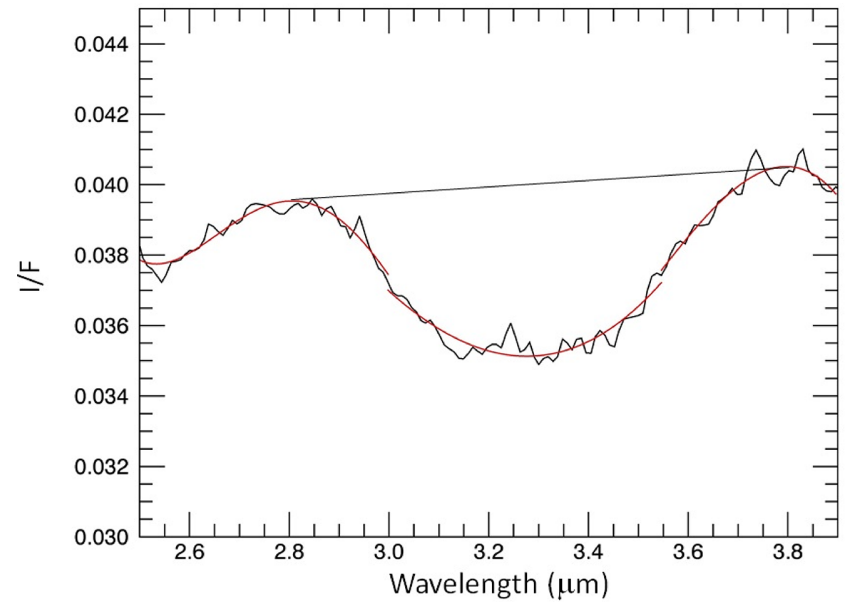

Figure 3. Definition of the $3.2 \mu \mathrm{m}$ band. The three red curves are the modelled left shoulder, reflectance trough, and right shoulder, respectively. The black straight line is the modelled band continuum.

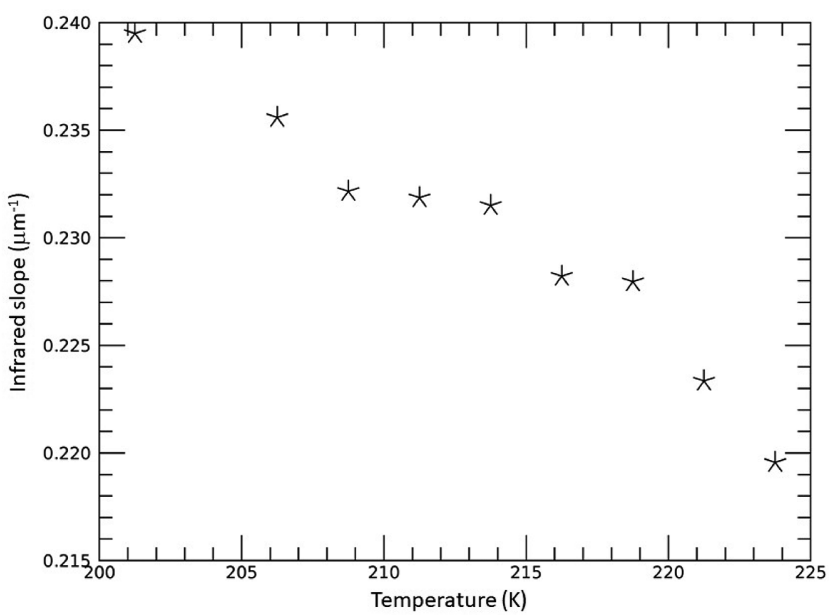

Figure 4. Babi region: an example of decreasing spectral slope with increasing temperature.

amount of water ice induced by weak cometary activity. Since spectral slopes are affected more than band centre shifts by small variations of water ice content (Ciarniello et al. 2016), in this work we analysed the behaviour of both the $3.2 \mu \mathrm{m}$ band centre $(\mathrm{BC})$ and the infrared spectral slope $(S)$ at varying temperature. The increasing amount of water ice would cause a BC shortward shift and $S$ decrease (i.e. flattening). If water ice is exposed from cometary activity, we should observe these spectral changes at higher temperatures (Fig. 2).

We defined BC and $S$ following Longobardo et al. (2017a). For the $3.2 \mu \mathrm{m}$ absorption band: the left shoulder is defined by a 4 th order polynomial fit computed between 2.5 and $3.0 \mu \mathrm{m}$; the reflectance trough is defined by a 3 th order polynomial fit between 3.0 and 3.55 $\mu \mathrm{m}$; and the right shoulder is defined by a 4th order polynomial fit between 3.55 and $3.9 \mu \mathrm{m}$ (Fig. 3). The band continuum is a linear fit between left and right shoulder maxima, whereas the band centre corresponds to the reflectance minimum after the continuum removal. The band depth $\mathrm{BD}$ is computed as

$\mathrm{BD}=1-R_{c} / R_{\mathrm{con}}$,

where $R_{c}$ and $R_{\text {con }}$ are the $I / F$ and the continuum at the band centre (Clark \& Roush 1984). The $3.2 \mu \mathrm{m}$ band depth does not need a photometric correction, being phase-independent (Longobardo et al. 2017a) and was used to select the spectra on the basis of their signal-to-noise ratio (see Section 2).

The infrared slope was defined as

$S=\frac{\left(R_{2.0}-R_{1.1}\right)}{R_{1.1}(2.0-1.1)}$,

where $R_{\lambda}$ is the radiance factor at the wavelength $\lambda$, expressed in microns. The slope was photometrically corrected by applying the relation

$S=S_{0}-b \varphi$,

where $S_{0}$ and $S$ are the uncorrected and corrected slope (in $\mu \mathrm{m}^{-1}$ ), respectively, $\varphi$ is the phase angle (in degrees) and $b=1.48 \times 10^{-4}$ $\mu \mathrm{m}^{-1} \mathrm{deg}^{-1}$ (Longobardo et al. 2017a).

Temperatures were calculated from the thermal radiance measured in the infrared spectral range of $4.5-5.1 \mu \mathrm{m}$ by means of a Bayesian approach (Tosi et al., under revision).

\subsection{GIADA}

Whereas the GDS-only and GDS + IS detections give a direct speed measurement, the IS measures the particle momentum $p$. However, Della Corte et al. (2016a) used the GDS + IS measurements to obtain the following empirical relation to retrieve speed also for IS-only detections

$v=A m^{\gamma}$, 
Table 1. Band centre (BC) shift and infrared slope $(S)$ decrease (in $\mathrm{nm}$ and $\mathrm{mm}^{-1}$, respectively) as a function of temperature $(T)$ in the geomorphological regions of the 67P's Northern hemisphere. For each region, the corresponding macro-region and temperature coverage (as observed by VIRTIS) are also reported.

\begin{tabular}{lcccc}
\hline Region & Macro-region & $T$ excursion $(\mathrm{K})$ & BC shift $(\mathrm{nm})$ & $S$ decrease $\left(\mathrm{mm}^{-1}\right)$ \\
\hline Aker & Body & $210-225$ & 8 & 12 \\
Anubis & Body & N/A & N/A & N/A \\
Apis & Body & N/A & 10 & N/A \\
Ash & Body & $180-225$ & 10 & 10 \\
Aten & Body & $205-225$ & 7 & 14 \\
Babi & Body & $200-225$ & 10 & 17 \\
Khephry & Body & $190-225$ & 6 & 13 \\
Imhotep & Bottom & $190-225$ & 3 & 4 \\
Bastet & Head & $218-224$ & 2 & 5 \\
Hathor & Head & $215-225$ & 6 & 6 \\
Hatmeith & Head & $212-225$ & 0 & 19 \\
Ma'at & Head & $190-225$ & 5 & 5 \\
Maftet & Head & $220-225$ & 3 & 6 \\
Nut & Head & $220-225$ & 3 & 6 \\
Serget & Head & $205-225$ & 4 & 10 \\
Anuket & Neck & $210-225$ & N/A & N/A \\
Atum & Neck & N/A & 15 & 16 \\
Hapi & Neck & $210-225$ & 9 & 18 \\
Seth & Neck & $190-225$ & & \\
\hline
\end{tabular}

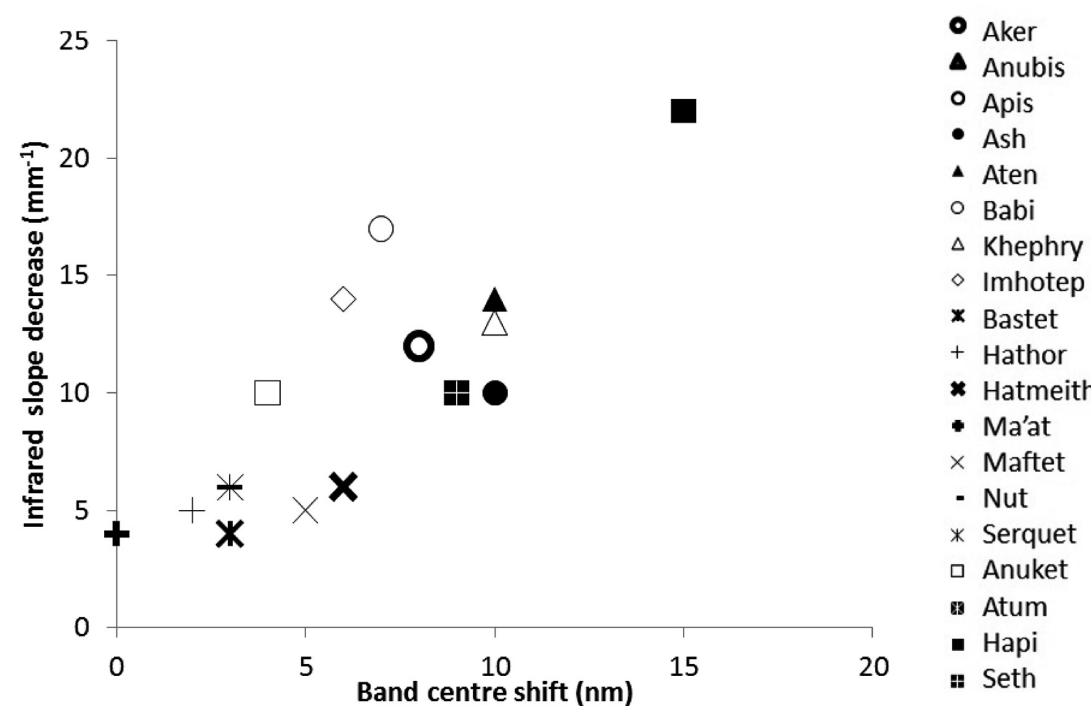

Figure 5. Infrared slope decreases as a function of band centre shift with temperature. Each symbol corresponds to a geomorphological region: in particular, circles and triangles to body, diamond to bottom, mathematical symbols to head, and squares to neck regions.

where $v$ is the particle speed, $m=p / v$ is the particle mass, $A$ and $\gamma$ are parameters depending on the heliocentric distance and on the phase angle. We applied this empirical relation to the entire GIADA data set.

GIADA calibration activity performed with comet dust analogues (Ferrari et al. 2014; Della Corte et al. 2016b) and dust modelling (Fulle et al. 2015) assessed that the type of detection depends on the following particle physical properties:

(i) GDS + IS and IS-only detections are due to compact particles (Della Corte et al. 2015).

(ii) Dust showers, i.e. ensembles of numerous GDS-only detections clustered in space and in time (duration up to $30 \mathrm{~s}$ ), are ascribed to fragmentation of fluffy parent particles (Fulle et al. 2015). (iii) Isolated GDS-only detections are associated with compact particles that, due to their trajectory, did not impact on the IS.

In this paper, we associated dust showers with fluffy particles, assuming that each shower is generated by fragmentation of a single parent particle, and all the other detections with compact particles.

\section{METHOD}

\subsection{VIRTIS}

We studied the BC and $S$ parameters dependence on temperature in all the geomorphological regions of $67 \mathrm{P}$ observed in the considered inbound arc. Their concurrent decrease could indicate exposition 

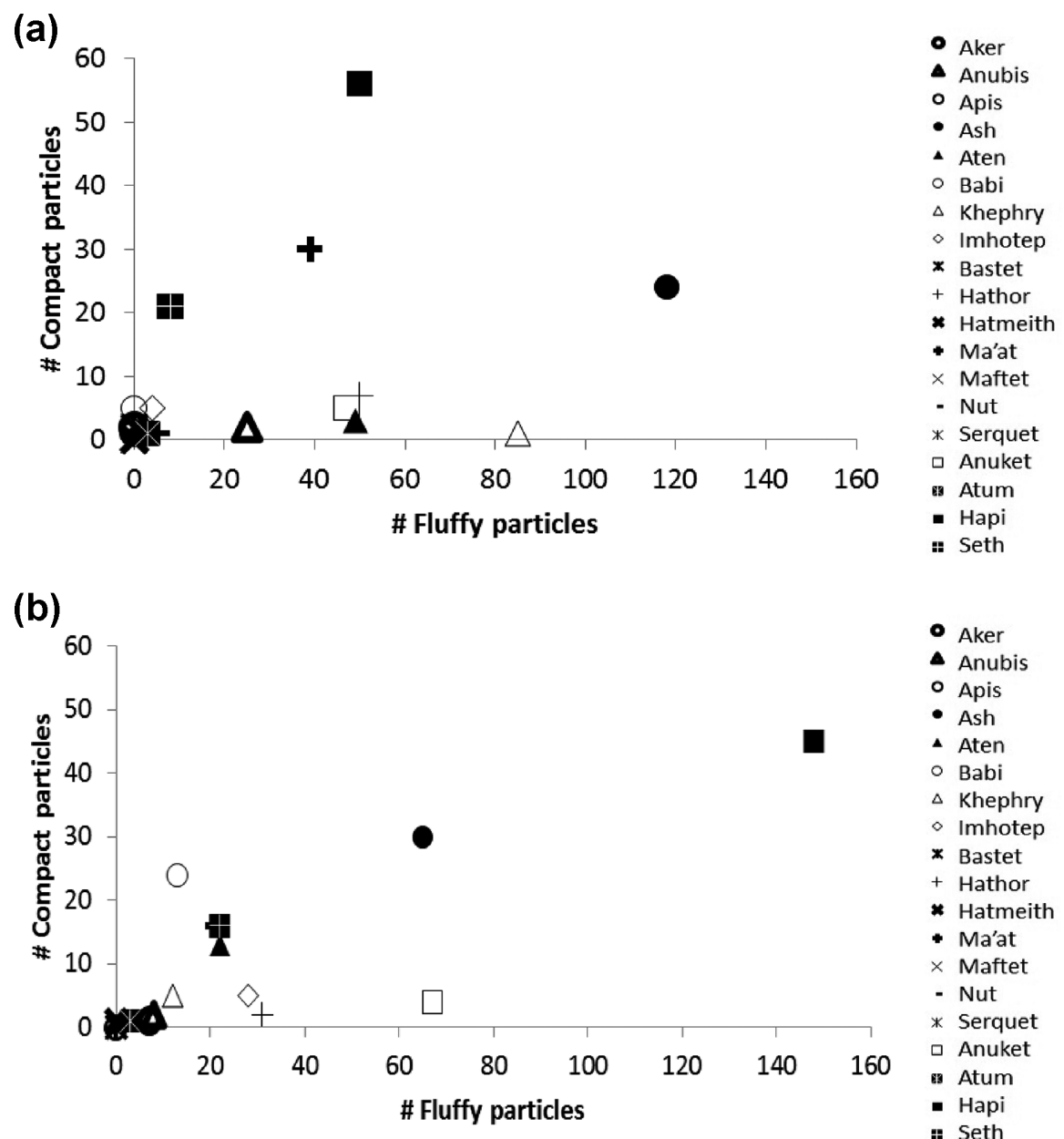

- Aker
A Anubis
- Apis
- Ash
A Aten
- Babi
$\triangle$ Khephry
- Imhotep
* Bastet
+ Hathor
* Hatmeith
* Ma'at
× Maftet
- Nut
* Serquet
- Anuket
a Atum
- Hapi
* Seth

Figure 6. Correlation between the number of fluffy fragments versus compact particles as detected by GIADA in the coma (top) and after the application of the traceback algorithm, i.e. on the nucleus surface (bottom). Each symbol indicates the geomorphological region ejecting the dust particles (nucleus case, bottom plot) or the region on which the coordinates of the coma detection are projected (coma case, top plot): in particular, circles and triangles to body, diamond to bottom, mathematical symbols to head, and squares to neck regions.

of water ice and hence can be considered an indicator of cometary activity.

We defined temperature bins of $2.5 \mathrm{~K}$ width, and we calculated the average band centre and the average infrared slope in each bin for every region. We studied the behaviour of these average values with temperature, observing three different cases:

1. The coverage of temperature excursion for the region is not wide enough $(<15 \mathrm{~K})$ to identify a trend. This occurs for Anubis, Apis, and Atum regions, which were excluded from VIRTIS data analysis.

2. The spectral parameter (BC and/or $S$ ) decreases with increasing temperature (see Fig. 4). In this case we calculated the parameter change as the difference between the parameter values at the lowest and at the highest temperatures of the region.

3. The spectral parameter (BC and/or $S$ ) does not show any trend with the temperature. In this case, we assigned a zero value to the corresponding parameter change. This case occurs only once and concerns the $\mathrm{BC}$ behaviour in Ma' at region.

Finally, we related the $\mathrm{BC}$ and $S$ change, in order to check for a possible correlation. The correlation was evaluated by means of the Pearson coefficient, already used in the spectral analysis of planetary surfaces (e.g. Longobardo et al. 2017b), defined as
$\sigma_{F B} / \sigma_{F} \sigma_{B}$, being $\sigma_{F}, \sigma_{B}$, and $\sigma_{F B}$ the variance of the two sets of parameters and their covariance, respectively. A Pearson coefficient indicates strong correlation if larger than 0.7 , moderate correlation if comprised between 0.4 and 0.7 , lack of correlation if smaller than 0.4 .

\subsection{GIADA}

An indicator of dust cometary activity can be defined as the number of particles ejected by a certain region/area. Therefore, we developed an algorithm, based on particle speed measured by GIADA, to reconstruct the motion of each dust particle detected in the coma and tracing it back to the surface, taking into account the spacecraft distance from the nucleus and the comet rotation.

A specific analysis was performed in the case of dust showers, applying the following procedure:

a. Definition of the shower. A shower is defined by all particles detected at a temporal distance lower than one second with respect to the previous detection.

b. Selection of shower's detection. We removed the detections with measuring speeds less than $0.3 \mathrm{~m} \mathrm{~s}^{-1}$, corresponding to fragments disturbed by the spacecraft potential (Fulle et al. 2015), and 


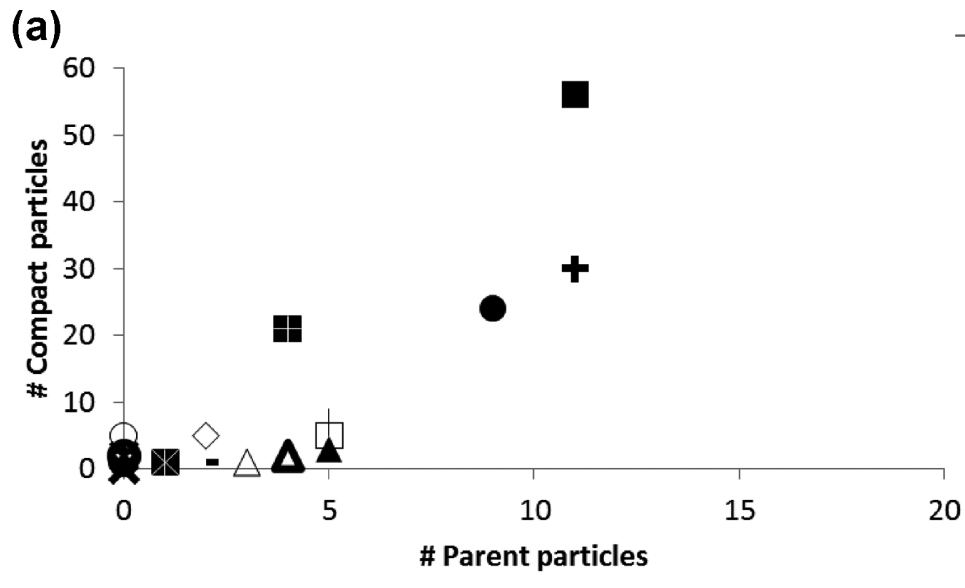

- Aker

- Anubis

- Apis

- Ash

- Aten

- Babi

$\Delta$ Khephry

- Imhotep

* Bastet

+ Hathor

* Hatmeith

- Ma'at

$\times$ Maftet

- Nut

* Serquet

- Anuket

Atum

- Hapi

* Seth

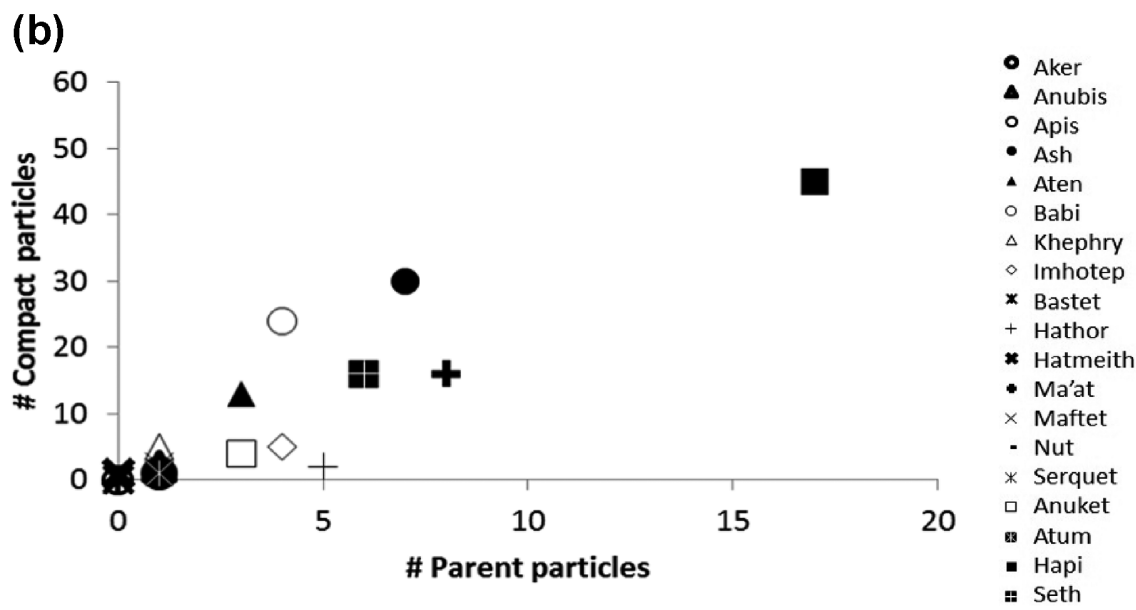

Figure 7. Correlation between the number of fluffy parent versus compact particles as detected by GIADA in the coma (top) and after the application of the traceback algorithm, i.e. on the nucleus surface (bottom). Each symbol indicates the geomorphological region ejecting the dust particles (nucleus case, bottom plot) or the region on which the coordinates of the coma detection are projected (coma case, top plot): in particular, circles and triangles to body, diamond to bottom, mathematical symbols to head, and squares to neck regions.

greater than $45 \mathrm{~m} \mathrm{~s}^{-1}$, corresponding to poorly reliable measurements due to GDS system behaviour (Della Corte et al. 2016b).

c. Retrieval of the parent particle's speed.

Case 1: The shower includes a particle detected by GDS + IS, thus its speed is assumed to be parent particle's speed.

Case 2: The shower consists of GDS-only detections. A speed histogram with bins of $5 \mathrm{~m} \mathrm{~s}^{-1}$ is built, and the median speed in the most populated bin is assumed to be representative of the entire shower speed, as the parent particle's speed.

Even though in the shower speed distribution particle speeds greater than $9 \mathrm{~m} \mathrm{~s}^{-1}$ could be less reliable (Fulle et al. 2015), we included these detections in our procedure as they are not statistically significant; therefore, their inclusion does not change our conclusions (see Section 6).

To reconstruct the particle's motion back to the nucleus and identify the geomorphological region from which each particle detected by GIADA was ejected, we made the following assumptions:

1. The motion is radial from the nucleus to GIADA. This assumption is partially corroborated by the results obtained by Della Corte et al. (2016a)

2. The motion is uniformly accelerated up to a distance of $11 \mathrm{~km}$ from the surface and then rectilinear uniform. This assumption is based on coma dust models (Ivanovski et al. 2017; Zakharov et al. 2018).

From these assumptions, and by means of the algorithm,

(i) We calculated the particle time of flight from the nucleus surface to GIADA and quantified the comet rotation relative to this time interval.

(ii) By combining time of flight and comet rotation, we identified the geomorphological region from which each particle was ejected.

We performed a correlation analysis by means of the Pearson coefficient (see Section 4.1), before and after the application of the traceback algorithm, between: (1) the number of fluffy fragments and compact particles standing above (in the case of coma) or ejected (in the case of nucleus) from each region; (2) the number of parent and compact particles standing above (in the case of coma) or ejected (in the case of nucleus) from each region. Since the number of fluffy fragments is also dependent on the fragmentation process of the parent particles, the number of parents is more closely related to the actual emission of fluffy dust particles. 

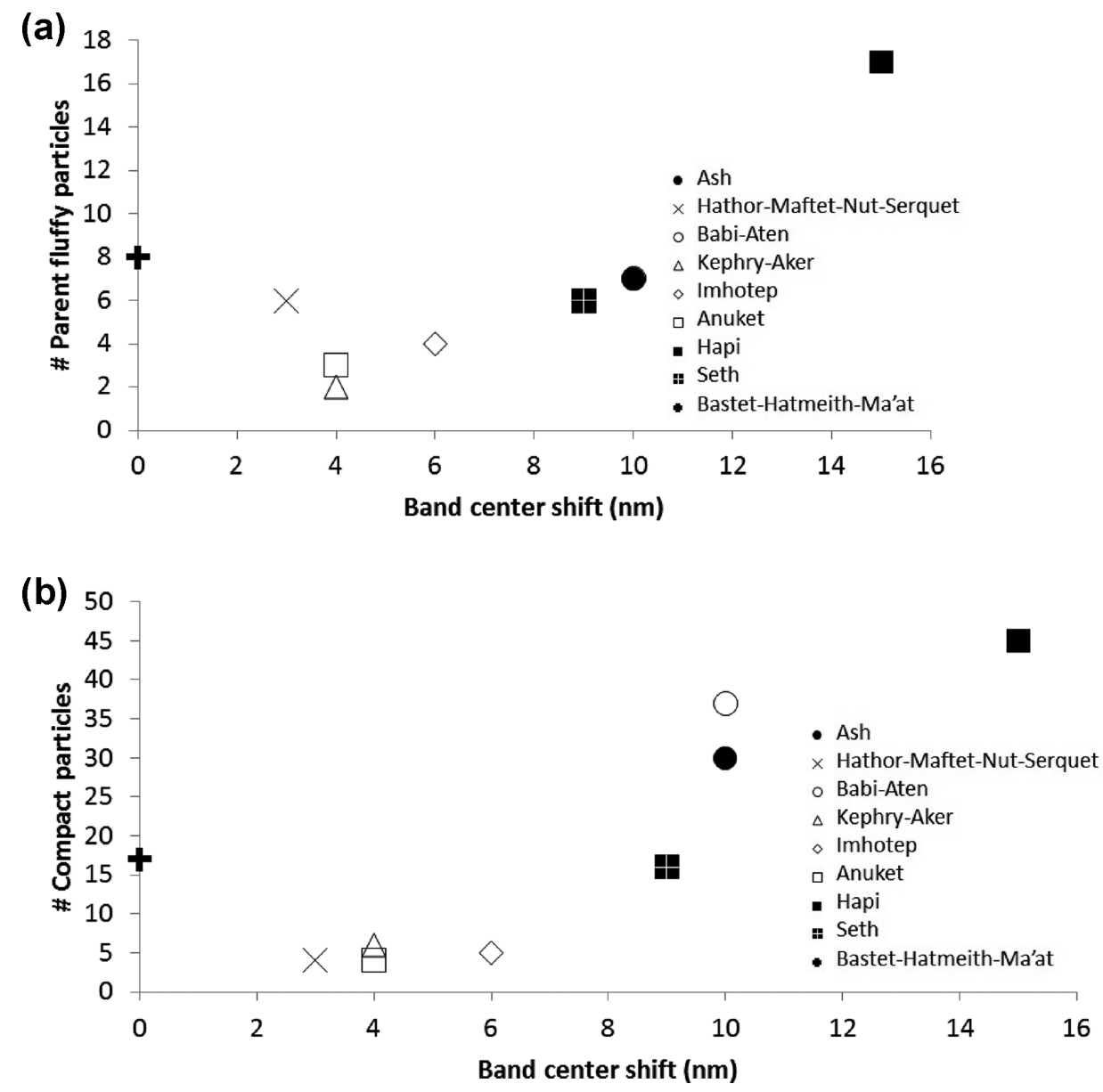

Figure 8. Number of fluffy parent (top) and compact (bottom) particles ejected from each nucleus region as a function of the BC shift measured by VIRTIS. Each symbol corresponds to a geomorphological region: in particular, circles and triangles to body, diamond to bottom, mathematical symbols to head, and squares to neck regions.

\subsection{GIADA-VIRTIS data fusion}

To date, we suggested that the $\mathrm{BC}$ and $S$ decrease at increasing temperature, on the VIRTIS side, and the number of parent and compact particles ejected, on the GIADA side, are indicators of cometary activity. To confirm this hypothesis, we related them by means of a Pearson analysis.

Since the GIADA indicators (number of ejected particles) also depend on the region's area (especially for small regions) and the VIRTIS indicators do not, for a reliable comparison, we merged some regions in order to have an extension of at least 3000 $\mathrm{deg}^{2}$, i.e. Bastet-Hatmeith-Ma'at, Babi-Aten, Kephry-Aker, and Hathor-Maftet-Nut-Serget. The Anubis, Atum, and Apis regions, that were not studied with VIRTIS, were excluded from the data fusion analysis, even if appearing to be active from a GIADA indicator.

\section{RESULTS}

\subsection{VIRTIS}

The BC shift and $S$ decrease in each of the studied regions together with the coverage of temperature excursion for each region are reported in Table 1 and in Fig. 5. The Pearson coefficient between the $\mathrm{BC}$ and $S$ changes is 0.8 , indicating high correlation. The changes are larger in the 67P neck (Hapi, Seth) and body (Ash, Kephry, Babi, and Aten) macro-regions, and lower in the head (Ma'at, Bastet, Nut, Serget, Hathor). Even there is not a trend between spectral parameters change and temperature excursion, regions with a lowtemperature variation $(<10-15 \mathrm{~K})$ are generally associated with lower BC shift and $S$ decrease.

\subsection{GIADA}

The correlation of the spatial distribution between fluffy fragments and compact particles in the coma and on the surface, i.e. before and after the application of the traceback algorithm, is shown in Fig. 6. After having applied the traceback procedure, the Pearson coefficient increases from 0.4 (weak/moderate correlation) to 0.8 (high correlation).

The relation between fluffy parent and compact particles in the coma and on the surface, i.e. before and after the application of the traceback algorithm, is shown in Fig. 7. After applying the traceback procedure, the Pearson coefficient increases from 0.8 (high correlation) to 0.9 (very high correlation).

GIADA detected a higher dust activity from Hapi and Seth (neck), Ash (body), and Ma'at (head) regions, which are also the more extended ones. 

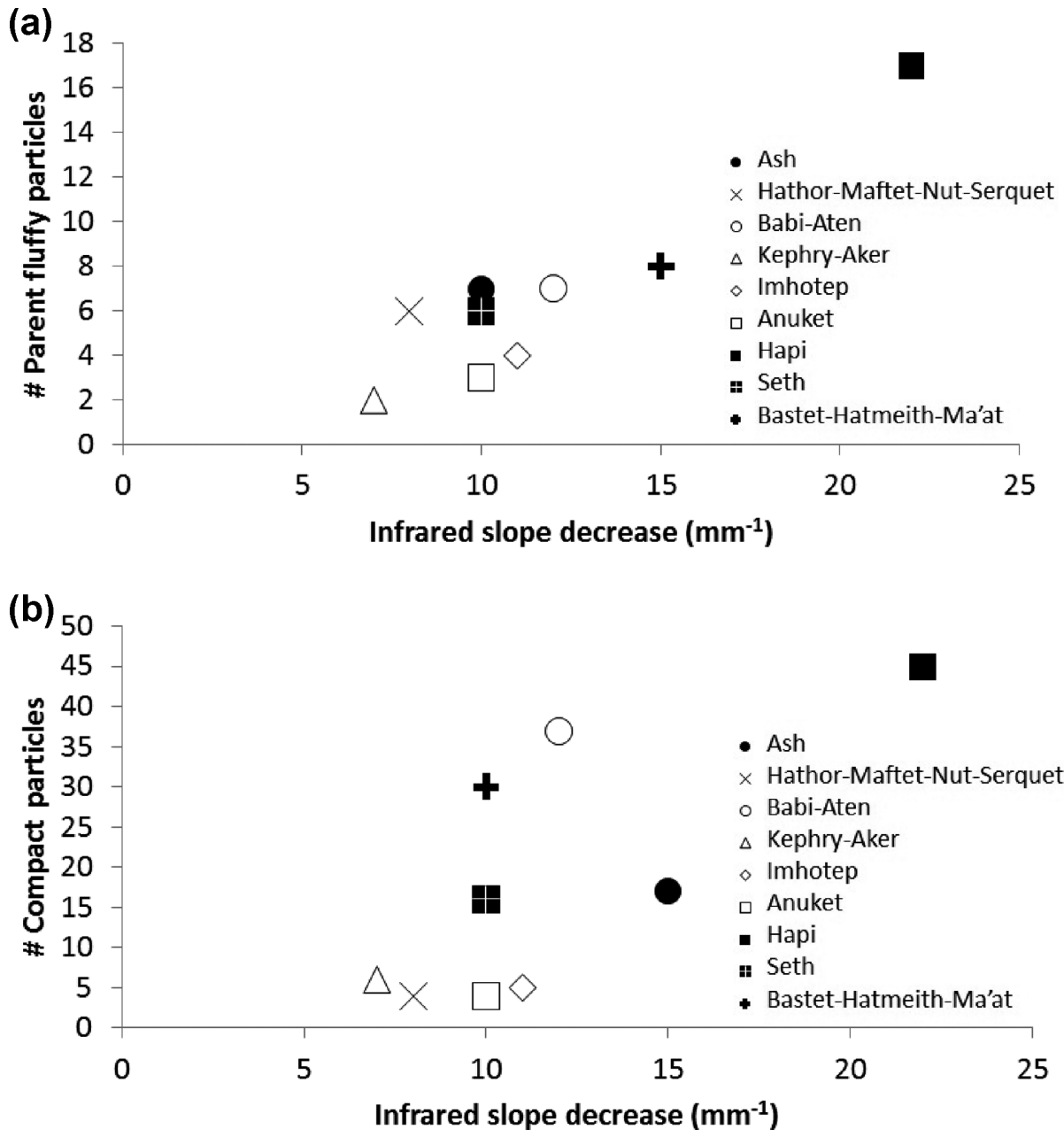

Figure 9. Number of fluffy parent (top) and compact (bottom) particles ejected from each nucleus region as a function of the $S$ decrease measured by VIRTIS Each symbol corresponds to a geomorphological region: in particular, circles, and triangles to body, diamond to bottom, mathematical symbols to head, and squares to neck regions.

\subsection{GIADA-VIRTIS data fusion}

We compare here the $\mathrm{BC}$ and $S$ changes as a function of the temperature measured by VIRTIS with the number of parent and compact particles detected by GIADA and ejected from each of the studied $67 \mathrm{P}$ regions.

In Fig. 8, the BC shift is plotted with respect to the number of parent and compact particles. The corresponding Pearson coefficients are 0.7 for parent fluffy particles and 0.8 for the compact ones, indicating high correlations. According to both scatterplots, the most active regions are located in the neck (Hapi and Septh) and in the body (Ash and Babi-Aten). The Bastet-Hatmeith-Ma' at region is slightly outside of the general trend, showing a high number of ejected dust particles not corresponding to a significant BC shift.

Fig. 9 shows the number of parent and compact particles ejected from each nucleus region as a function of the infrared slope decrease with temperature. The corresponding Pearson coefficients are 0.9 and 0.7 , indicating a very high and high correlation, respectively. We can conclude that the most active regions are Hapi, Babi-Aten, Seth, and Ash, but also the merged region BastetHatmeith-Ma'at.

\section{DISCUSSION}

The high correlation between the band centre shift and infrared slope decrease (Fig. 5) suggests that the process inducing these two effects is the same. Exposition of water ice causes both a shortening of BC and a flattening of $S$. Thus, it is plausible that BC and $S$ changes are evidence of the connection among the surface temperature increase in the studied mission phase, the exposition of underlying water ice, and the cometary activity arise. This hypothesis was suggested by Longobardo et al. (2017a) based on the temperature trend of band centre observed for the 67P/Churyumov-Gerasimenko average and for a few specific geomorphological regions. The results obtained with this study, i.e. the high correlation between $\mathrm{BC}$ and $S$ changes with the temperature in all the geomorphological regions of the $67 \mathrm{P}$ Northern hemisphere, reinforces this hypothesis.

The high correlation that we find between specific areas of ejection of fluffy parent and compact particles indicates that they have common sources on 67P surface. After ejection, the two particle types disperse, as demonstrated by the low or absent correlation of their spatial distribution in the coma and as already observed by Della Corte et al. (2015). In fact, the high Pearson coefficient (i.e. 0.7) measured in the scatterplot of parent versus compact particles 
(a)

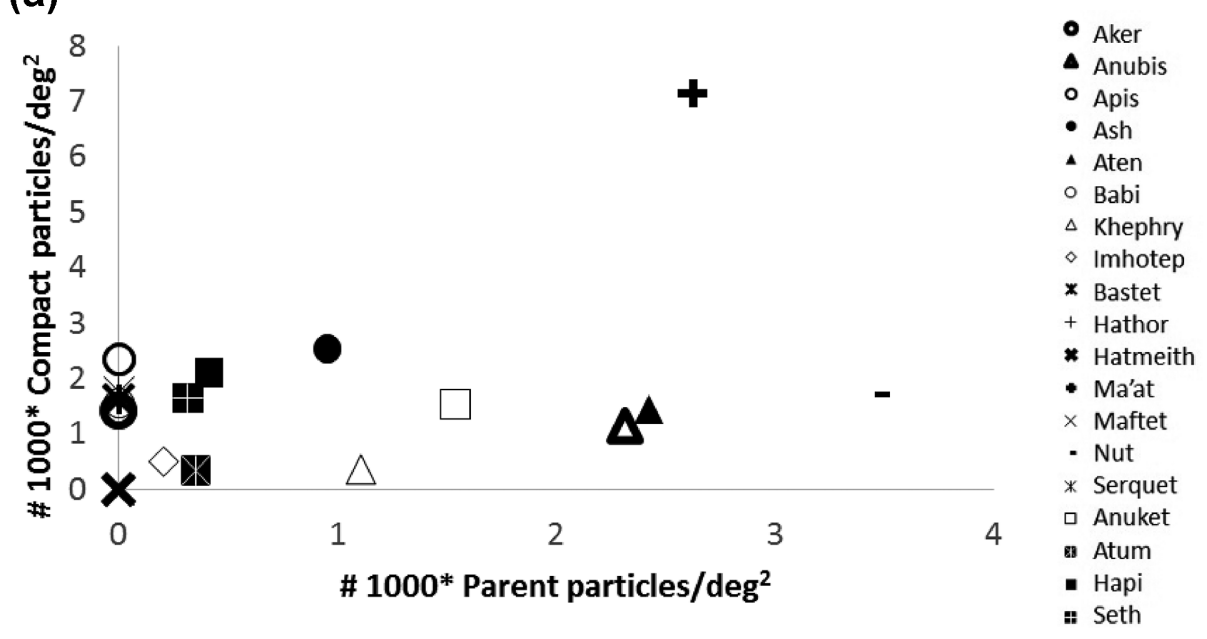

(b)

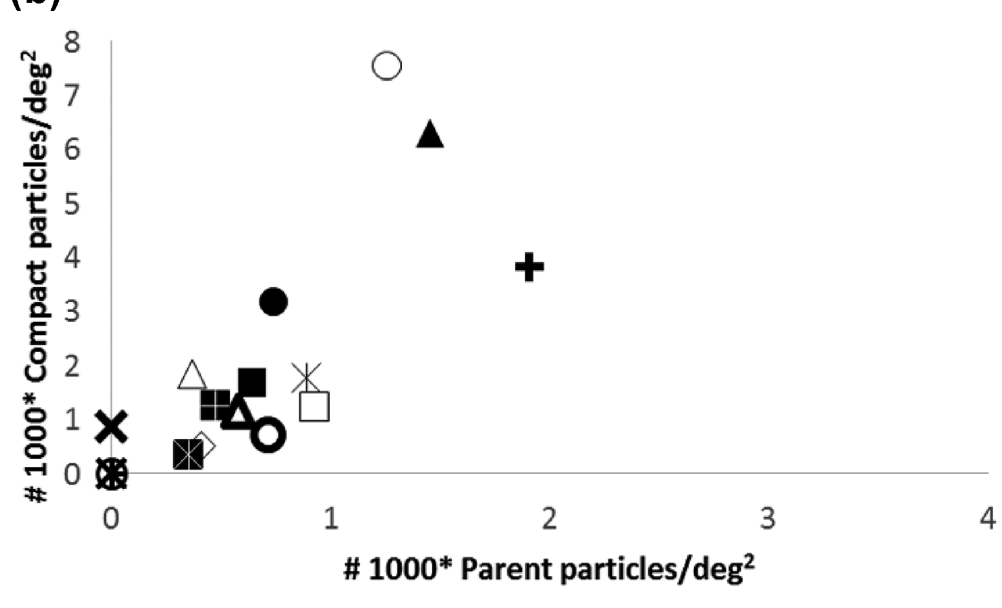

- Aker

- Anubis

- Apis

- Ash

- Aten

- Babi

$\triangle$ Khephry

- Imhotep

* Bastet

+ Hathor

* Hatmeith

- Ma'at

$\times$ Maftet

- Nut

* Serquet

口 Anuket

* Atum

- Hapi

* Seth

Figure 10. Number of parent fluffy particles revealed per unit area (in square degrees) by GIADA before (top) and after (bottom) the traceback algorithm, i.e. in the coma and in the nucleus, respectively. Each symbol indicates the geomorphological region ejecting the dust particles (nucleus case, bottom plot) or the region on which the coordinates of the coma detection are projected (coma case, top plot): in particular, circles and triangles to body, diamond to bottom, mathematical symbols to head, and squares to neck regions.

in the coma (Fig. 7, top), i.e. before the application of the traceback algorithm, is observed because larger regions obviously emit a larger number of particles. Otherwise, if we analyse the number of emitted particles for unit area, we obtain a Pearson coefficient of 0.2 before traceback, i.e. an absence of correlation in the coma (Fig. 10, top), and 0.7 after trace back, i.e. high correlation on the nucleus surface (Fig. 10, bottom).

Therefore, fluffy parent and compact particles are ejected contemporarily from the same nucleus surface areas and then their spatial distribution is lost in the coma, due to their different velocities.

The high correlation between the GIADA and VIRTIS indicators (Figs 8 and 9) provides the definitive link between dust cometary activity and exposition of water ice on the surface. The most active dust-emitting regions are characterized by spectra showing the most prominent water ice signatures at high temperatures. Only the Bastet-Hatmeith-Ma' at region seems to be an exception. This finding might be due to an uncertainty, given by the occurrence of particles ejected from this region having velocities larger than $9 \mathrm{~m} \mathrm{~s}^{-1}$, which are the least reliable measurements. These velocities are retrieved only in the Bastet-Hatmeith-Ma'at region, which this could explain its anomalous behaviour. When excluding this region from the analysis, the Pearson coefficient between dust particles and $\mathrm{BC}$ shift is 0.9 , both for fluffy parents and compact, instead of 0.7 and 0.8, respectively. In addition, excluding Bastet-HatmeithMa' at region, the Pearson coefficients between $S$ decrease and compact particles increases from 0.7 to 0.8 . Interestingly, Poch et al. (2016) found a similar behaviour by simulating cometary activity (i.e. ice sublimation) on ice-tholins mixtures; after the formation of a crust layer, ejection of particles led to exposition of water ice and to blueing of mixture spectra's slope.

Based on the cometary activity indicators we considered, the most active regions of $67 \mathrm{P}$ during the studied mission phase are located in the neck (Hapi and Seth) and in the body (Ash, Babi, and Aten), whereas the bottom (Imhotep) and especially the head regions are the less active. This result is in agreement with the strong Hapi activity observed by various Rosetta instruments (e.g. De Sanctis et al. 2015; Tubiana et al. 2015; Hu et al. 2017). Moreover, Migliorini et al. (2017) found that the most active regions before perihelion, in terms of water vapour emission, are Hapi-Seth and Babi-Aten. 

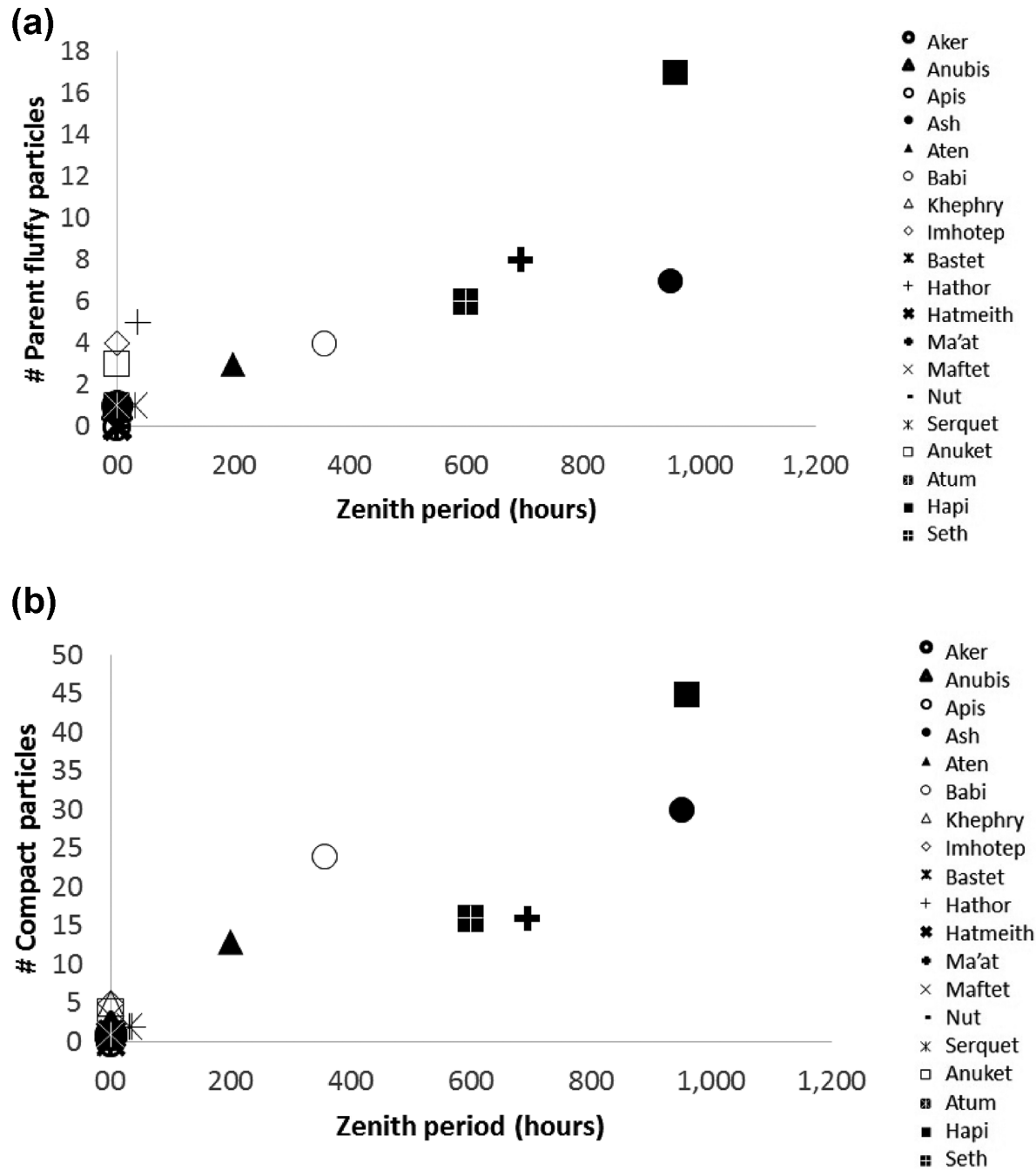

Figure 11. Number of ejected compact and fluffy parent particles as a function of Zenith period. Each symbol corresponds to a geomorphological region: in particular, circles and triangles to body, diamond to bottom, mathematical symbols to head, and squares to neck regions.

Their result is in agreement with the correlation between dust and water vapour emission found by Rinaldi et al. (2016), therefore we can conclude that exposition of water ice is caused by both water vapour and dust activity.

In order to evaluate the role of solar illumination on cometary activity, we related the insolation period with the number of fluffy parent and compact dust particles ejected from each region as retrieved from GIADA data. A cross-correlation between insolation period and BC and $S$ changes, measured by VIRTIS, would lead to the same conclusions, because of the correlation between GIADA and VIRTIS cometary activity indicators.

In Fig. 11, the number of parent and compact particles are plotted as a function of the Zenith period, i.e. the number of hours spent under normal illumination. We found a very strong correlation (Pearson coefficient is 0.9 ) between dust ejection and local insolation. The solar insolation is therefore the main driver of cometary activity, and therefore, it is unlikely that other drivers (internal heat) or properties (physical state of the surface) could affect the activity.

We conclude that dust ejection from the nucleus is strongly related to the illumination not only for compact particles but also of fluffy ones, which is different from what is observed in the coma (Della Corte et al. 2015, 2016a).

\section{CONCLUSIONS}

We studied 67P's activity in different geomorphological regions by combining VIRTIS and GIADA data.

On the VIRTIS side, we observed the change of the $3.2 \mu \mathrm{m}$ band centre and of the spectral slope between 1.1 and $1.9 \mu \mathrm{m}$, between the lowest and the highest temperature measured from each region. The obtained decreasing trends are related to exposition of water ice, due to dust release.

On the GIADA side, we developed a trace back algorithm (subsection 4.2), based on measured dust particle speeds, outcomes of coma dust models (Ivanovski et al. 2017; Zakharov et al. 2018) and comet rotation, to obtain the number of fluffy and compact particles ejected from each region.

We measured the correlation among all the retrieved VIRTIS and GIADA indicators of cometary activity, by means of the Pearson coefficient. These indicators were also compared to the illumination time of each morphological region. Table 2 summarizes all of the cross-correlation analyses performed.

Our combined GIADA and VIRTIS data analysis lead us to conclude the following: 


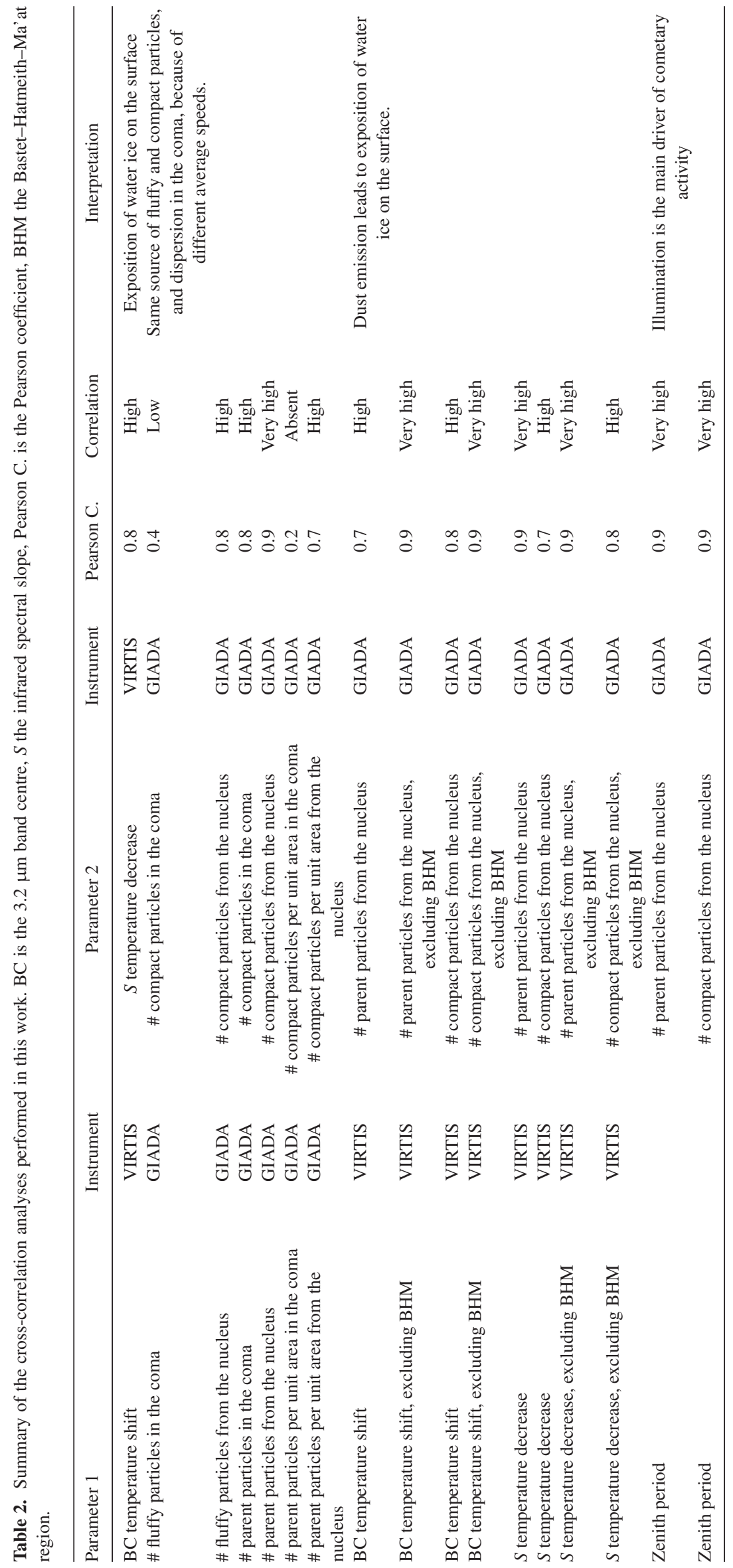


(i) The ejection of fluffy parent and compact particles is correlated on the nucleus but not in the coma: the two particles types are emitted contemporarily from common nucleus surface areas, and they are later spread during the motion due to their different velocities.

(ii) Dust emission determined by GIADA data is correlated to the BC and $S$ temperature changes measured by VIRTIS, reinforcing the link between cometary activity and the exposition of water ice (Longobardo et al. 2017a).

(iii) All cometary activity indicators identify the neck (Hapi, Seth) and the body (Ash, Babi, Aten) regions as the most active.

(iv) Cometary activity, as characterized by both GIADA and VIRTIS, and as already pointed out (e.g. Della Corte et al. 2015, 2016a), is strongly related to the insolation, which is the main driver, if not the only one.

\section{ACKNOWLEDGEMENTS}

GIADA (Grain Impact Analyser and Dust Accumulator) was built by a consortium led by the Universitá degli Studi di Napoli 'Parthenope' and INAF (Instituto Nazionale di AstroFisica) - Osservatorio Astronomico di Capodimonte, in collaboration with the Instituto de Astrofisica de Andalucia, Selex-ES, FI, and SENER. GIADA is presently managed and operated by Istituto di Astrofisica e Planetologia Spaziali-INAF, Italy. GIADA was funded and managed by the Agenzia Spaziale Italiana, with the support of the Spanish Ministry of Education and Science Ministerio de Educacion y Ciencias (MEC). GIADA was developed from a Principal Investigator proposal from the University of Kent; science and technology contributions were provided by CISAS, Italy; Laboratoire d'Astrophysique Spatiale, France; and institutions from the UK, Italy, France, Germany and the USA. Science support was provided by NASA through the U.S. Rosetta Project managed by the Jet Propulsion Laboratory/California Institute of Technology. We would like to thank A. Coradini for her contribution as a GIADA Co-Investigator. GIADA calibrated data will be available through ESA's Planetary Science Archive (PSA) website (http: //www.rssd.esa.int/index.php?project=PSA\&page=index). All data presented here are available on request before archiving in the PSA.

VIRTIS (Visible, InfraRed and Thermal Imaging Spectrometer) was built by a consortium formed by Italy, France, and Germany, under the scientific responsibility of the Istituto di Astrofisica e Planetologia Spaziali of INAF, Italy, which guides also the scientific operations. The consortium includes also the Laboratoire d'études spatiales et d'instrumentation en astrophysique of the Observatoire de Paris, France, and the Institut für Planetenforschung of DLR, Germany. The authors wish to thank the Rosetta Science Ground Segment and the Rosetta Mission Operations Centre for their continuous support. LVM acknowledges the DFG grant MO 3007/1-1.

This research was supported by the Italian Space Agency (ASI) within the ASI-INAF agreements I/032/05/0 and I/024/12/0.

\section{REFERENCES}

Barucci M. A. et al., 2016, A\&A, 595, 13

Capaccioni F. et al., 2015, Science, 347, 6220

Ciarniello M. et al., 2015, A\&A, 583, 14

Ciarniello M. et al., 2016, MNRAS, 462, S443

Clark R. N., Roush T. N., 1984, J. Geophys. Res., 89, 6329

Colangeli L. et al., 2007, Adv. Space Res., 39, 446

Coradini A. et al., 2007, Space Sci. Rev., 128, 529

Corte D. V. et al., 2014, J. Astron. Instrum., 3, 1350011

Della Corte V. et al., 2015, A\&A, 583, 10

Della Corte V. et al., 2016a, MNRAS, 462, S210

Della Corte V. et al., 2016b, Acta Astronaut., 126, 205

De Sanctis M. C. et al., 2015, Nature, 525, 500

El-Maarry M. R. et al., 2015, A\&A, 583, 28

El-Maarry M. R. et al., 2016, A\&A, 593, 20

Ferrari M. et al., 2014, Planet. Space Sci., 101, 53

Filacchione G. et al., 2016a, Nature, 529, 368

Filacchione G. et al., 2016b, Icarus, 274, 334

Filacchione G. et al., 2016c, Science, 354, 1563

Fulle M. et al., 2015, ApJ, 802, L5

Fulle M. et al., 2016, ApJ, 821, 14

Hu X. et al., 2017, A\&A, 604, 31

Ivanovski S. L., Zakharov V. V., Della Corte V., Crifo J. F., Rotundi A., Fulle M., 2017, Icarus, 282, 333

Keller H. U. et al., 2007, Space Sci. Rev., 128, 433

Longobardo A. et al., 2017a, MNRAS, 469, S346

Longobardo A. et al., 2017b, ICARUS, in press

Migliorini A. et al., 2017, A\&A, 589, 12

Poch O., Pommerol A., Jost B., Carrasco N., Szopa C., Thomas N., 2016, Icarus, 267, 154

Raponi A., 2014, Ph.D. dissertation, Università degli studi di Roma Tor Vergata,

Raponi A. et al., 2016, MNRAS, 462, S476

Rinaldi G. et al., 2016, MNRAS, 462, S547

Rotundi A. et al., 2015, Science, 347, aa3905

Sierks H. et al., 2015, Science, 347, aa1044

Thomas N. et al., 2015, Science, 347, 6220

Tubiana C. et al., 2015, A\&A, 573, 11

Zakharov V. V., Ivanovski S. L., Crifo J. F., Della Corte V., Rotundi A., Fulle M., 2018, Icarus, 312, 121

This paper has been typeset from a DOC file prepared by the author. 